\title{
Feature Extraction of Real-Time Image Using SIFT Algorithm
}

\author{
N. Sasikala, V. Swathipriya, M. Ashwini, V. Preethi, A. Pranavi, and M. Ranjith
}

\begin{abstract}
This paper deals with image processing and feature extraction. Feature extraction plays a vital role in the field of image processing. There exist different image pre-processing approaches for feature extraction such as binarization, thresholding, resizing, normalization so on...Then after these techniques are applied to obtain high clarity images. In Feature extraction object recognition and stereo matching are at the base of many computer vision problems. The descriptor generator module is changed for increasing the performance of algorithm. SIFT algorithm consist of two modules such as key point detection module and descriptor generation module. When compared to recent solution, the descriptor generation module speed is fifteen times faster and the time for feature extraction is also reduced.
\end{abstract}

Index Terms-SIFT (Scale invariant feature transform), SIFT HOG (Scale invariant feature transform histogram of oriented gradients), SURF (Speeded up robust features).

\section{INTRODUCTION}

The feature extraction is a particular form of dimensionality reduction in pattern recognition and image processing. From the original data obtaining the relevant information and representing that in lower dimensionality [16] space is the main aim of this paper. In feature extraction we will get image characteristics, a set of features which are important for feature classification. The extractions of these features are from initial set of measured data and builds derived values. Developing the effectiveness and efficiency of analysis and classification is the important role of feature extraction. The applications of this are robot navigation, panoramic photographs [14], stereo matching, object detection and recognition. This is useful where in the case of reducing the number of resources needed for processing without losing important information. To Detect and describe local features in digital images SIFT (scale invariant feature transform) is used.

\section{SURF (SPEEDED UP ROBUST FEATURES)}

SURF is a local descriptor [1] and detector. SURF is partially inspired by SIFT algorithm but it is severaltimes faster than that of SIFT descriptor. SURF enables to robust [12] against different image transformations [16].

In this algorithm in order to detect the interest points, it uses integer approximation of determinant of Hessian blob detector, in which pre-computation of integral image is performed.
In order to obtain the original image multi resolution pyramid technique is used and the shape of an image with same size and reduced bandwidth is copied. This gives a special blurring effect on the original image which is scale space, ensures that the point of interest are scale invariant.

SURF [7] algorithm is based on some principles which has three main parts,

\section{Interest point detection \\ 2.Local neighborhood description \\ 3.MATCHING.}

\section{SURF DESCRIPTOR:}

The aim of a descriptor is to provide unique description of the image features. For every point of interest, descriptors are computed in a local manner and hence description is obtained for earlier stored image. The descriptor dimensionality has a direct impact on both its computational complexity and point matching robustness[7]. A short descriptor provides more robust against appearance variation, but it does not have sufficient discrimination which may rise to false images.

The first step involves fixing a reproducible orientation according to information from the interest points. In accordance with the orientations obtained, a square region is constructed which extracts SURF descriptor from it.

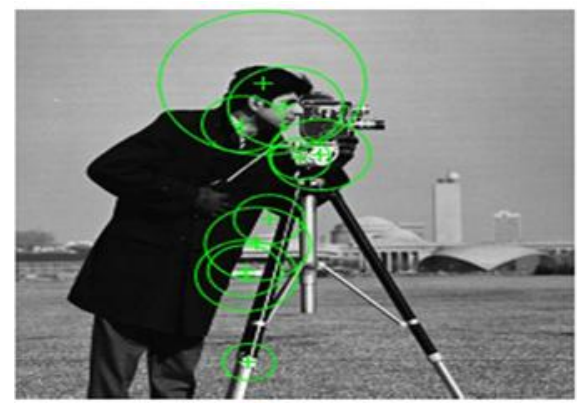

Fig.1. Detection of SURF features and SURF points identification

\section{SIFT HOG:}

SIFT HOG [2], or scale invariant feature transform histogram of oriented gradients is a feature descriptor which is used to extract features from the data provided.

The HOG descriptor completely focuses on shape of the object. HOG descriptor not only identifies the pixels in the edges but also shows the directions as well.

This process is done by using gradient and orientation [5] 
of the edges. Additionally, these orientations are calculated in localized portions [10]. The total image is divided into minor subparts and for each subpart gradient and orientations are calculated. Then at last the HOG would generate a histogram for each part separately. As the histogram is obtained from orientation and gradients it is named as histogram of oriented gradients.

HOG features can be created using the following steps: Step1: Preprocess the data of the stored image.

Step2: Calculating the gradients in two dimensions.

Step3: Calculate the magnitude and orientation.

Step4: Calculate the histogram of gradients in $8 * 8$ cells. Step5: Normalize gradients [13] in $16^{*} 16$ cells.

Step6: Features for the complete image.
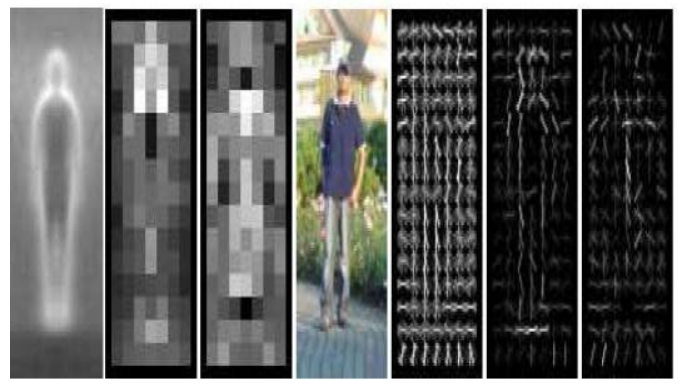

Fig. 2. Pictorial representation of HOG for human detection

$\checkmark \quad$ SIFT is used for identification of specific objects [12].

$\checkmark$ HOG is used for classification.

\section{SIFT (SCALE INVARIANT FEATURE TRANSFORM)}

SIFT [3] is the most proposed algorithm [11] due to its inherent properties such as invariance, changes in illumination [11] and 3D camera view point. There exist two different algorithms in SIFT descriptors. The first algorithm deals with problem of illumination variance.

The second algorithm is designed to tackle expression variations.

\section{SIFT DESCRIPTOR}

SIFT feature extraction method consists of four main steps,

* Scale extreme detection

* Removal of unreliable key points

* Orientation assignment and

* Key point descriptor calculation.

From the set of reference images SIFT key points of objects are extracted and are stored in the data base. When a new object is recognized then the image captured is verified with the images in the data base and finds whether the features are matched with the priorly stored information.

From the obtained set of matches, the orientation, scale, location is identified to get good matches. The probability of that particular set of features implies the presence of an object.

Feature description [4] of the object can be provided by extracting interesting points on the object.

This description obtained is used to predict the image of the object in same or different location.
The features in the training image can be detected even if there exist a change in the image scale, illumination and noise. As the features in the training image does not vary with the changes in the scale, illumination etc., so, it is called as invariant SIFT feature descriptor. SIFT features can essentially be applied to tasks which requires identification of matching [9] two images. SIFT is used for recognition of particular objects in 2D and 3D image reconstruction, robot localization, image panorama stitching and epipolar calibration.

\section{EXPERIMENTAL RESULTS}

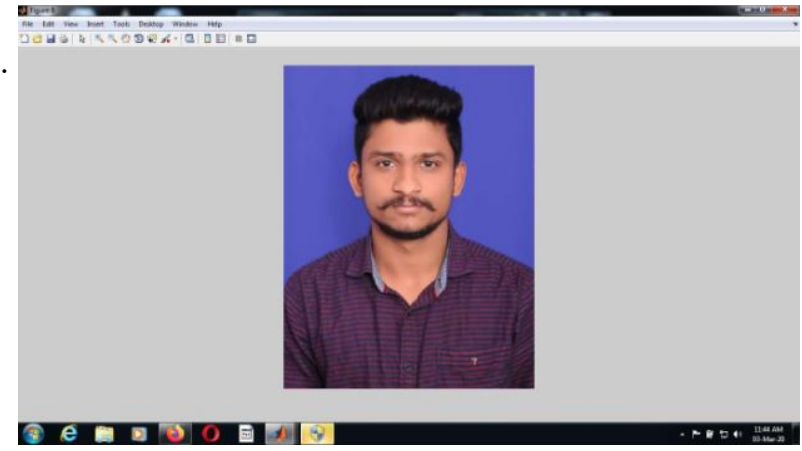

Fig. 3. Original Image Resized

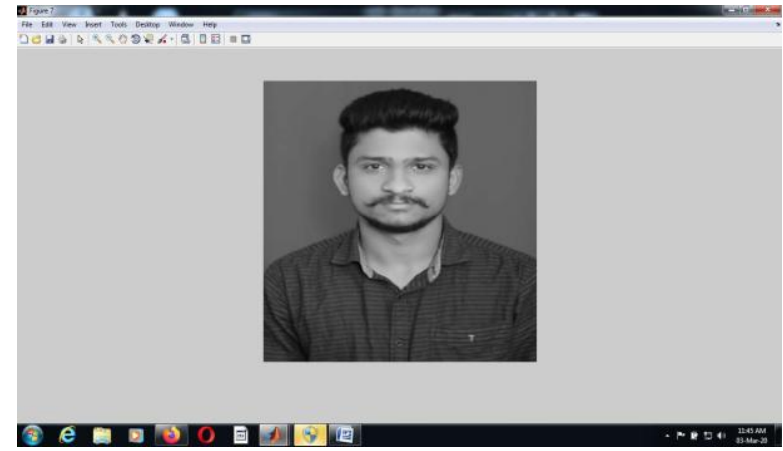

Fig. 4. RGB to Gray converted Image

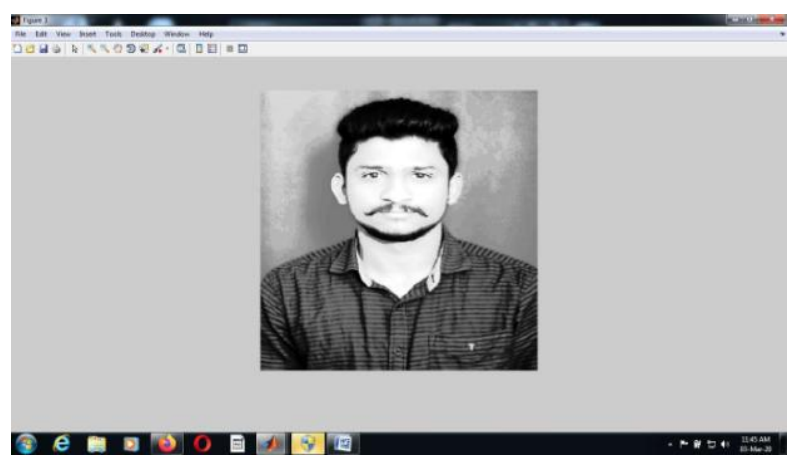

Fig. 5. Histogram Equalised Image 


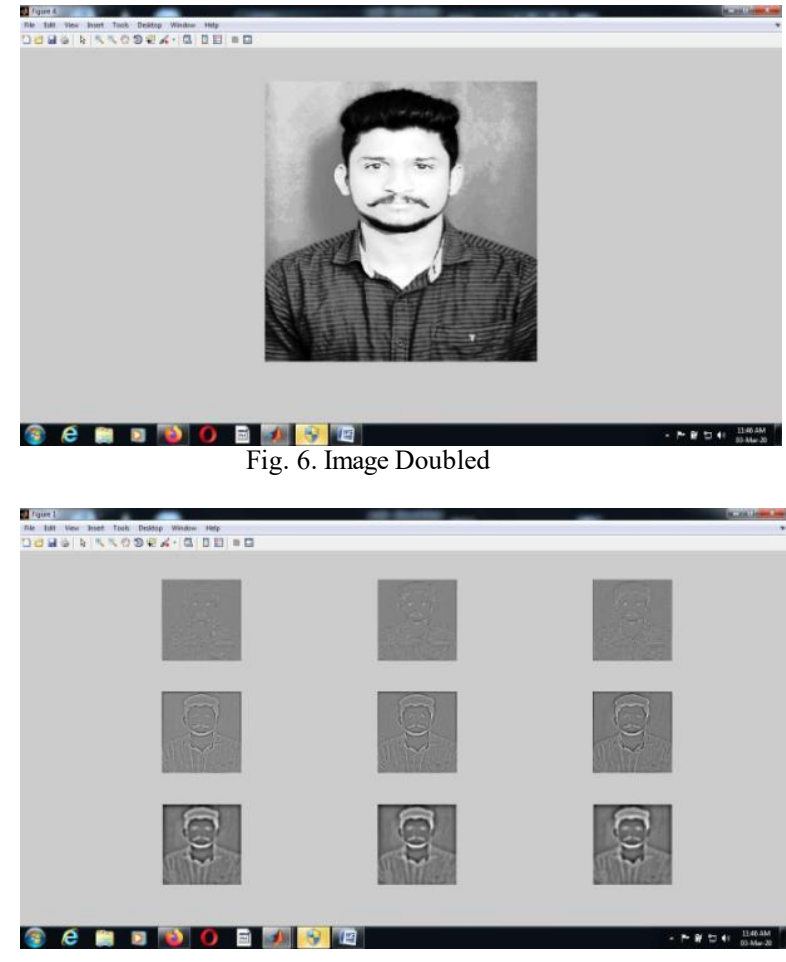

Fig. 7. Interpolation of original image ( eliminates the feature points of low contrast or poorly localised on an edge)

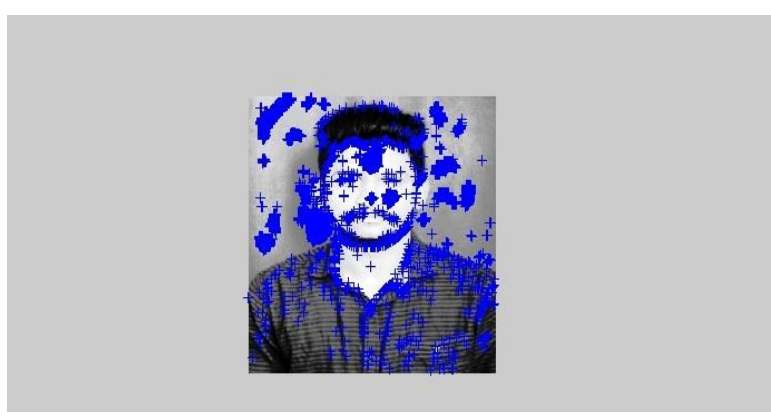

Fig. 8. Image detects unsearchable points and adds minor orientation points

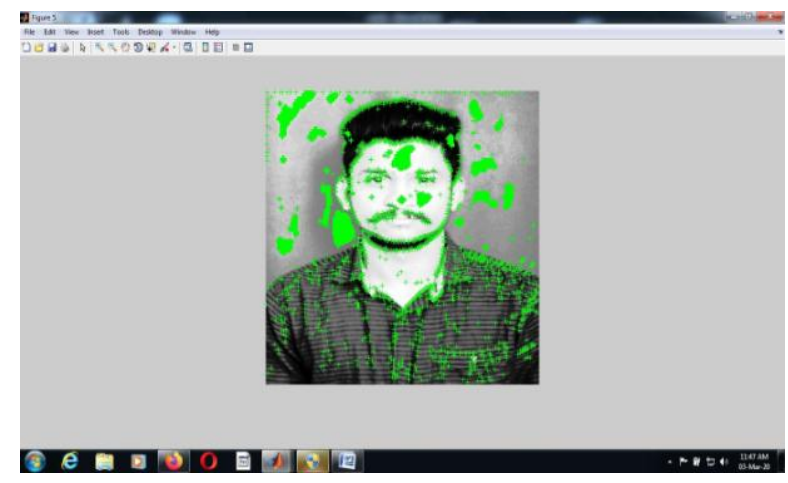

Fig. 9. Key point feature descriptor

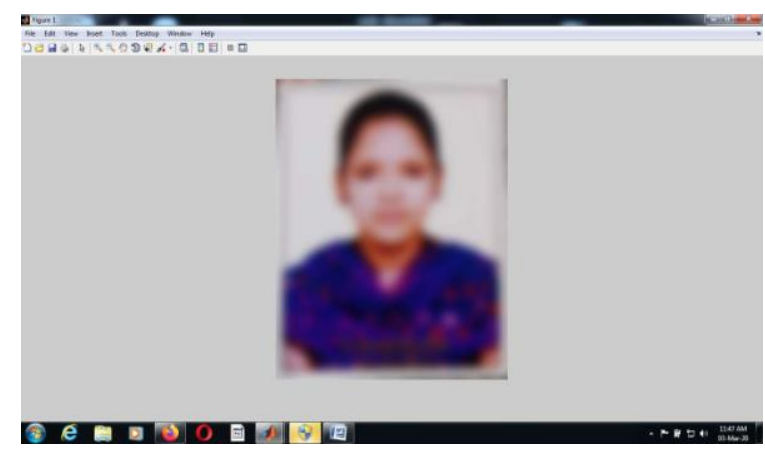

Fig. 10. Blurred Image

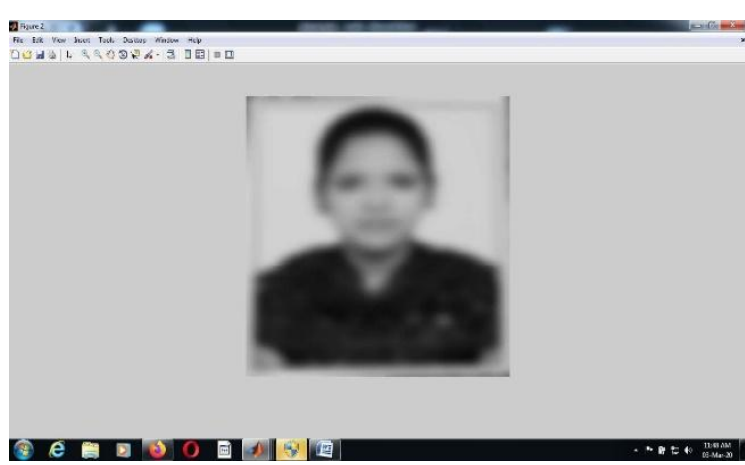

Fig. 11. RGB to Gray converted Image

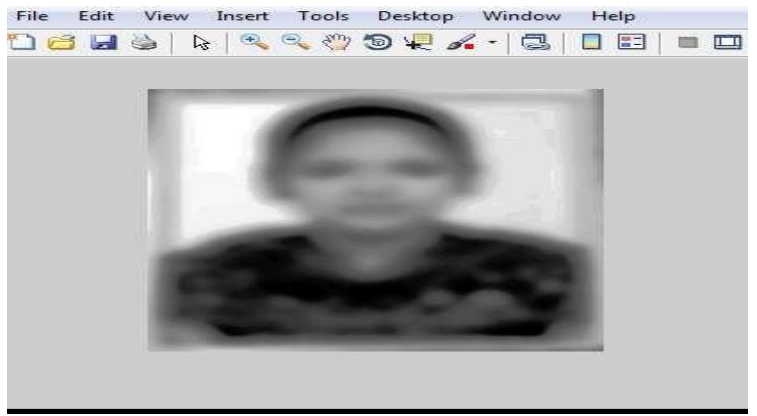

Fig. 12. Histogram Equalised Image

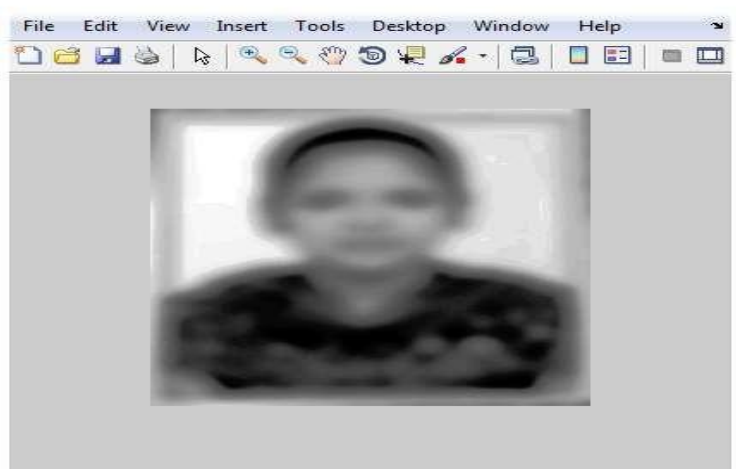

Fig. 13. Image Doubled 


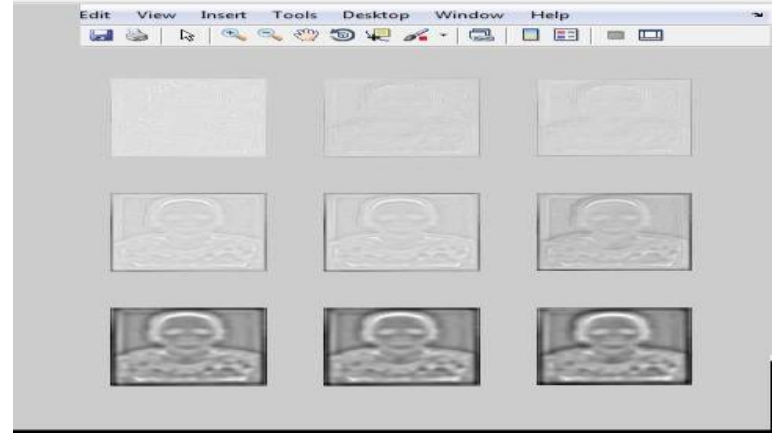

Fig. 14. Interpolation of original image (eliminates the feature points of low contrast or poorly localized on an edge)

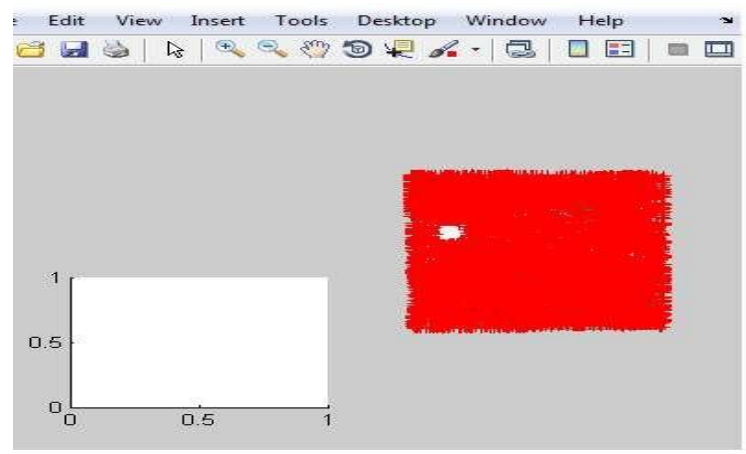

Fig. 15. Image detects unsearchable points and adds minor orientation points

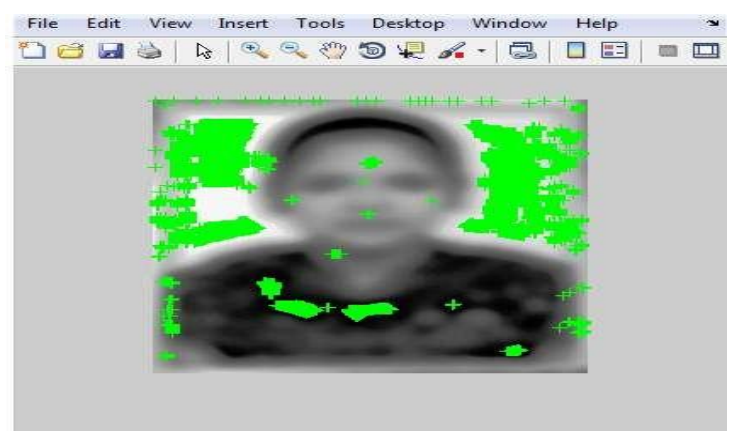

Fig. 16. Key point feature descriptor

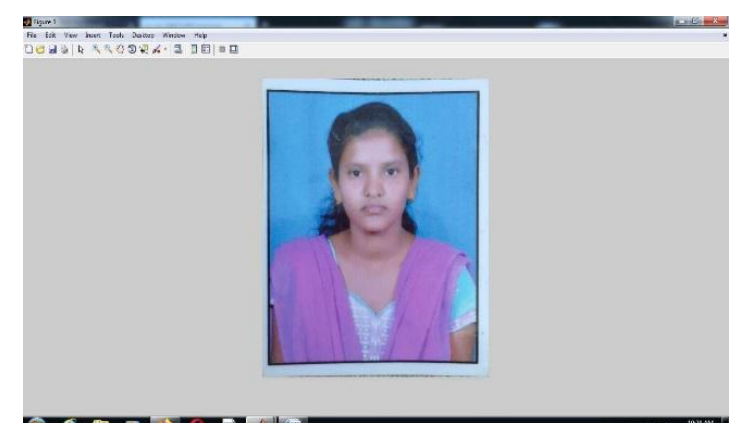

Fig. 17. Original Image Resized

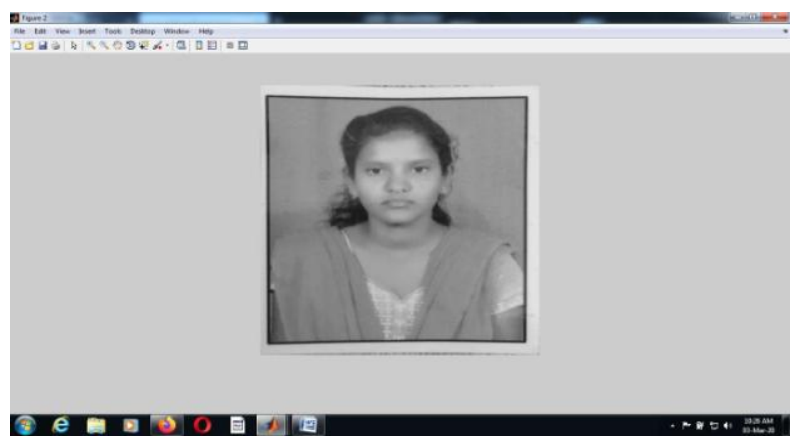

Fig .18. RGB to Gray converted Image

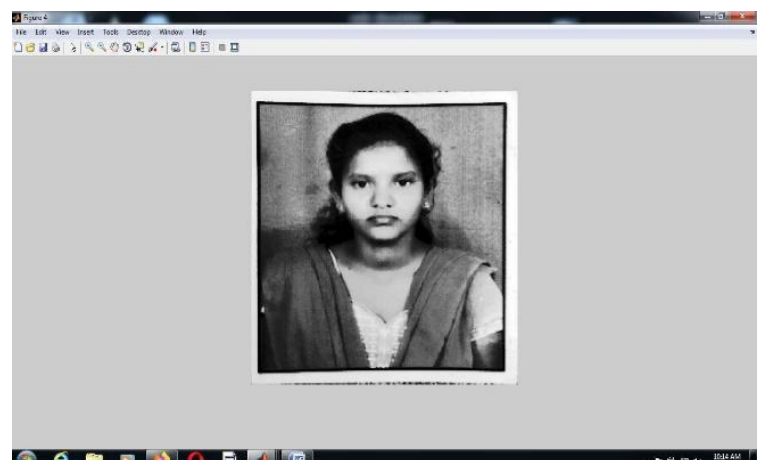

Fig. 19. Histogram Equalised Image

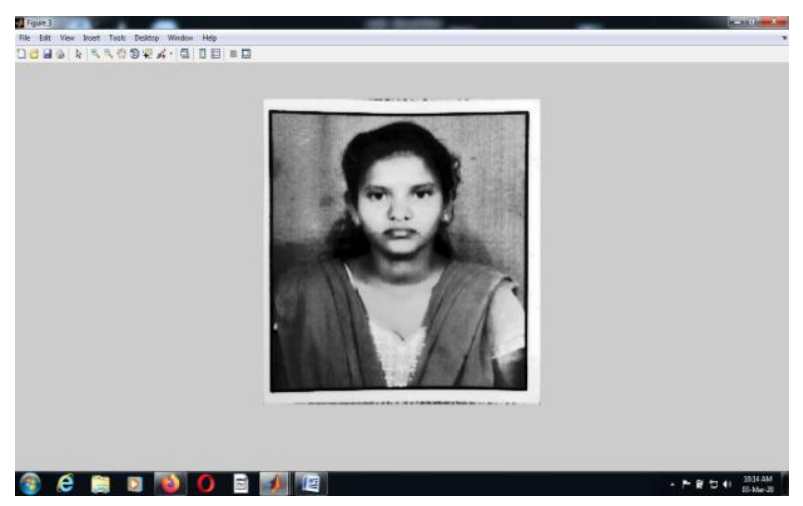

Fig. 20. Image Doubled

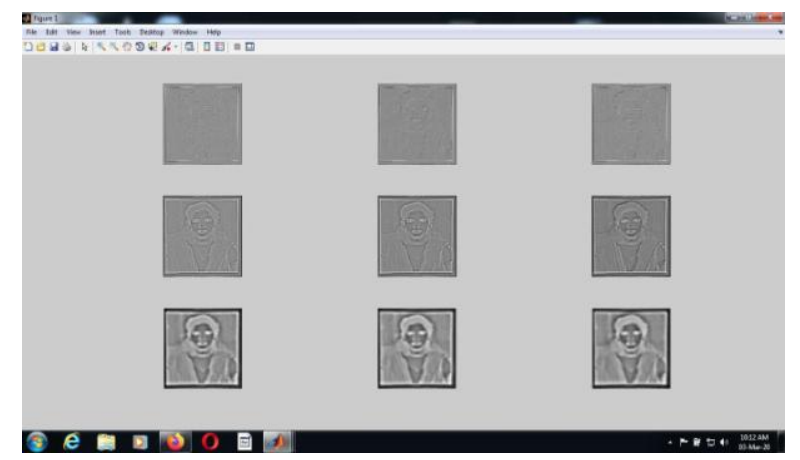

Fig. 21. Interpolation of original image ( eliminates the feature points of low contrast or poorly localised on an edge) 


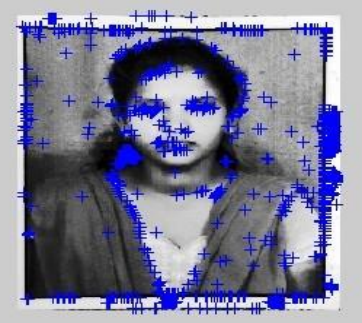

Fig. 22. Image detects unsearchable points and adds minor orientation points

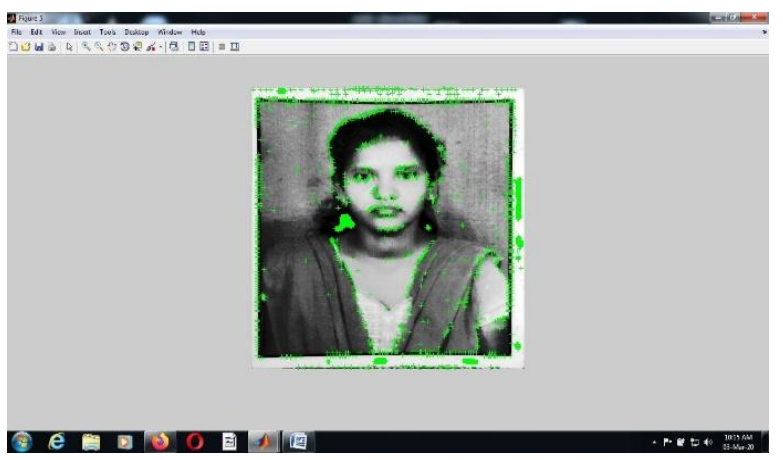

Fig 23.: Key point feature descriptor

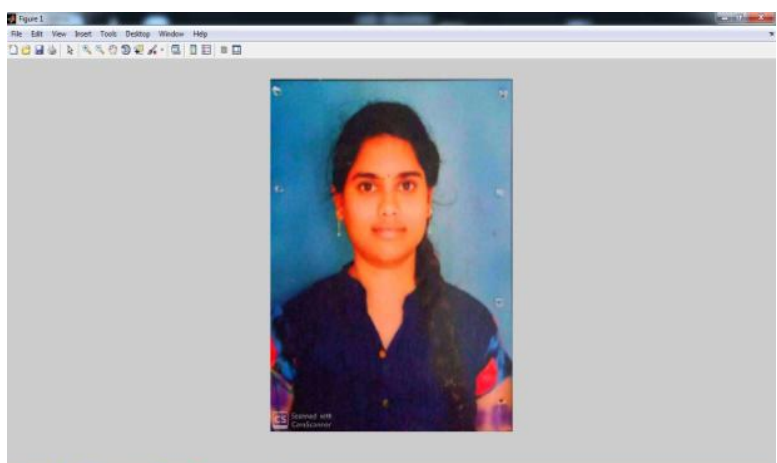

Cendorotir

$+\infty=0, \min$

Fig. 24. Increased saturation image Resized

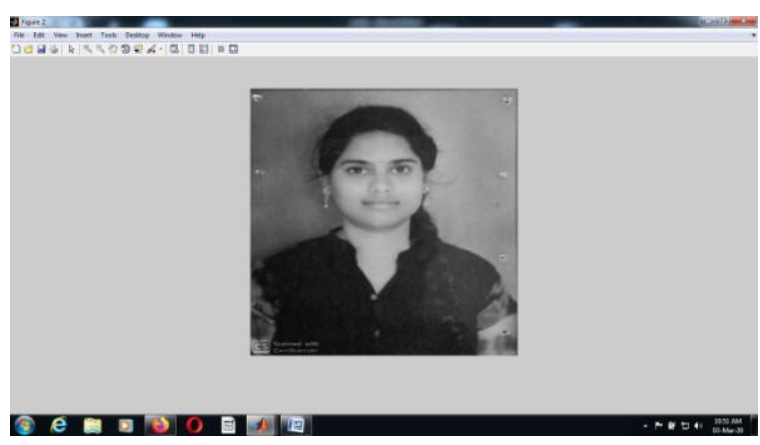

Fig. 25. RGB to gray converted image

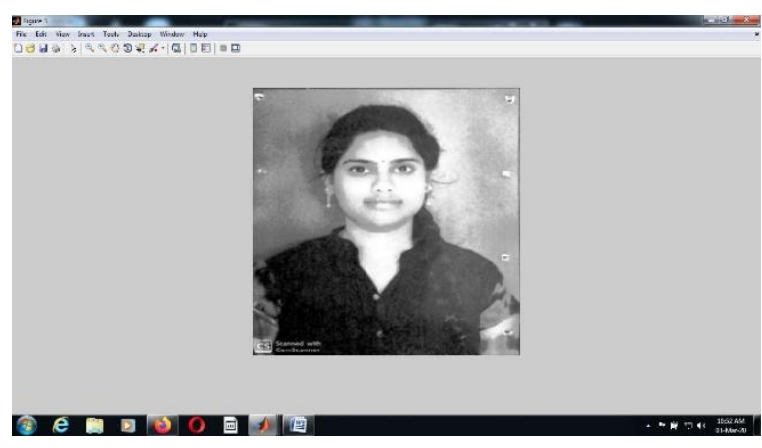

Fig. 26. Histogram Equalised Image

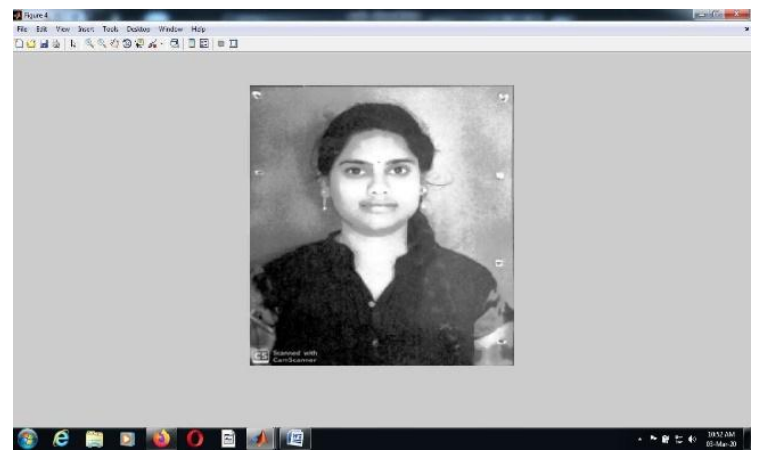

Fig. 27. Image Doubled

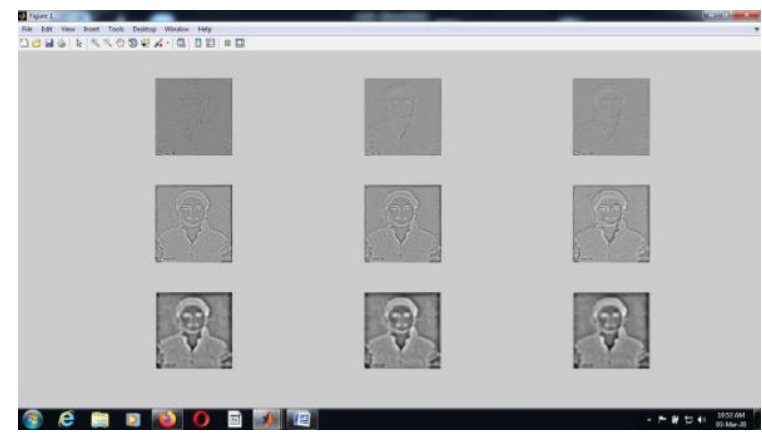

Fig. 28. Interpolation of original image (eliminates the feature points of low contrast or poorly localized on an edge)

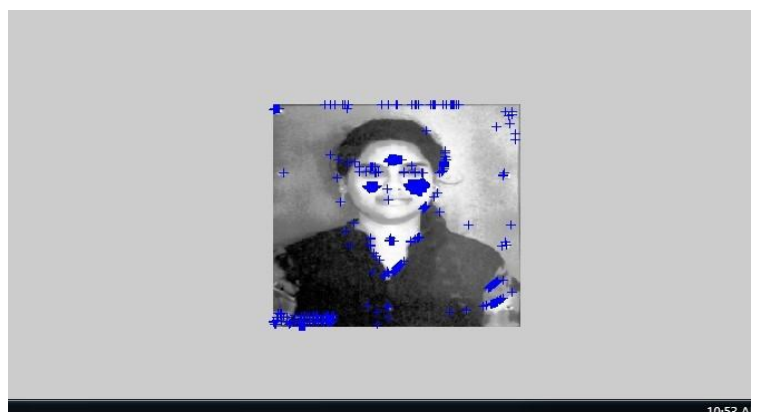

Fig 29. Image detects unsearchable points and adds minor orientation points 


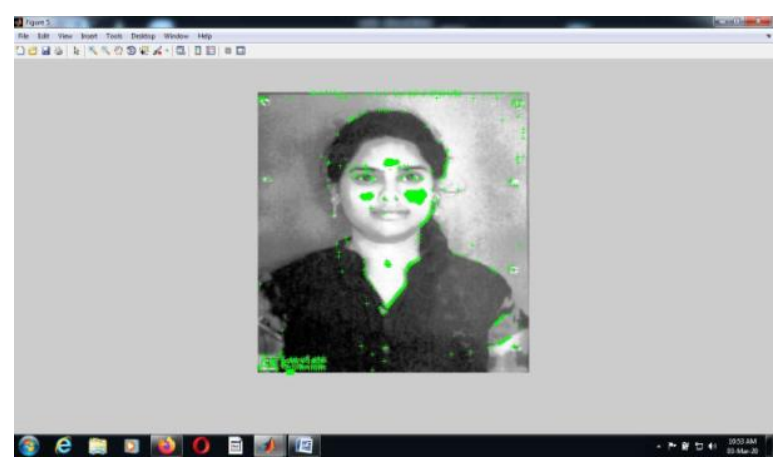

Fig. 30. Key point Feature descriptor

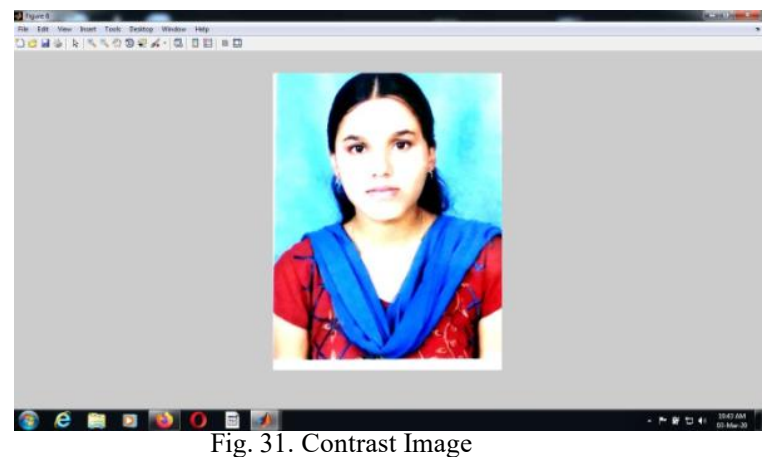

Fig. 31. Contrast Image

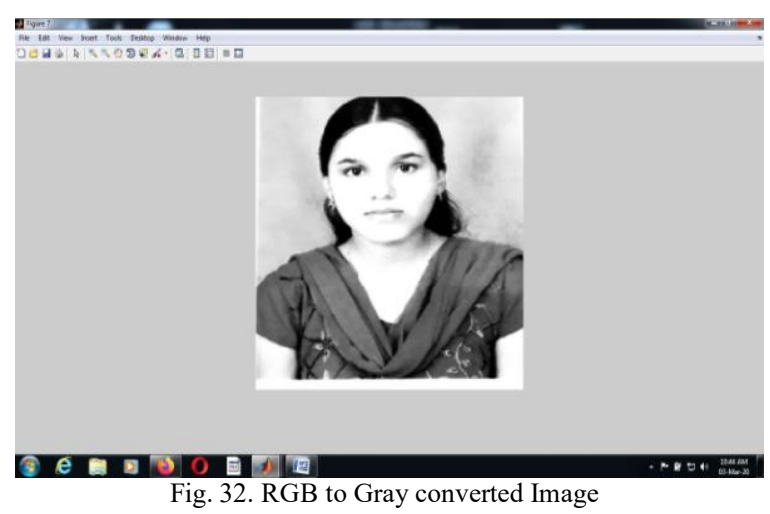

Fig. 32. RGB to Gray converted Image

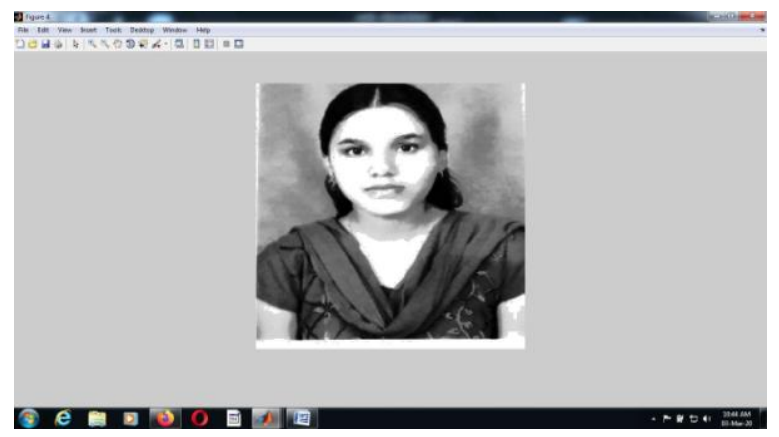

Fig. 33. Histogram Equalised Image

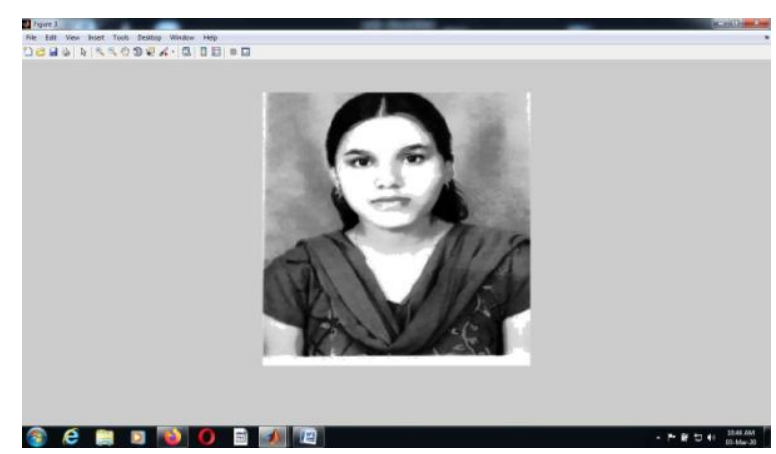

Fig. 34. Image Doubled

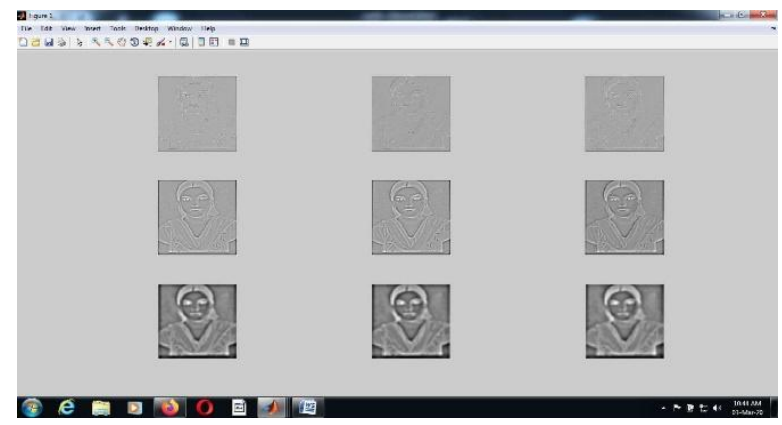

Fig. 35. Interpolation of original image (Eliminate the feature points of low contrast or poorly localized on an edge)

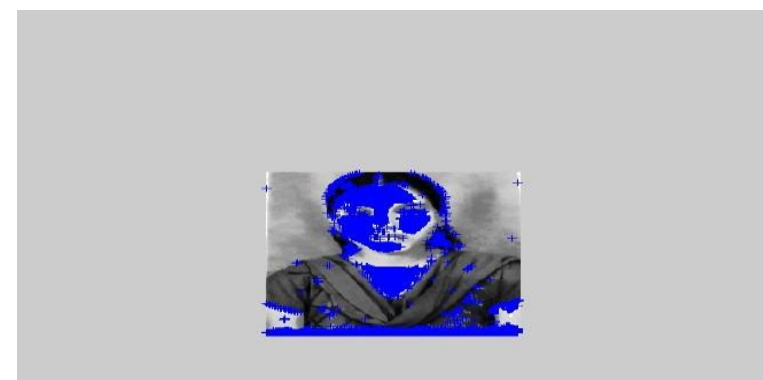

Fig. 36. Image detects unsearcable points and adds minor orientation points

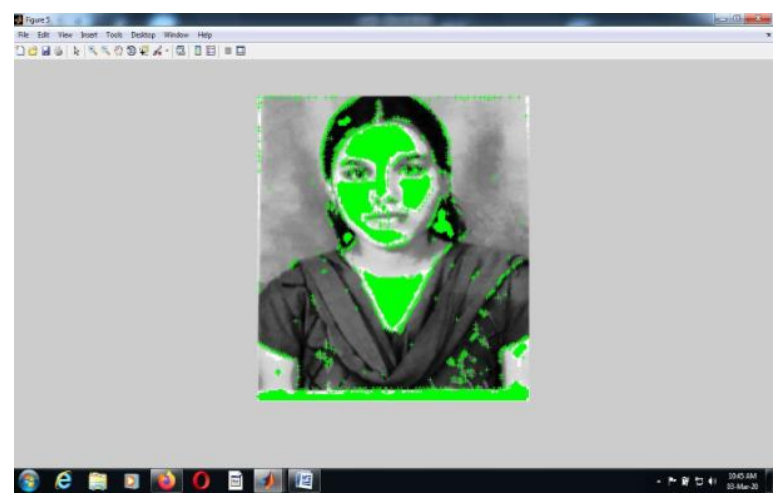

Fig. 37. Key point feature Descriptor 


\section{CONCLUSION}

Feature extraction technique is used to extract features without losing the original information. Along with simple features most advanced type of features are also extracted. Real time feature extraction is very important now a days as this is used in identifying systems.

The algorithm should be robust to noise [8] and should be speed in recognizing features.

The computation speed of the algorithm should be less so that it can be used in the modern gadgets and systems. Sift is on such algorithm that take strong features in the image and uses that to recognize.

Sift is robust to noise and computation [6] speed of the algorithm is less. This can be used in the identifying systems as well as in the security systems. Some applications of feature extraction are latent semantic analysis, data compression, data decomposition and projection, and pattern recognition. Feature extraction can also be used to enhance the features.

\section{REFERENCES}

[1] J.S. Patil, Dr.G. Pradeepini "An examination on restorative photograph evaluation the usage of photograph Descriptors frameworks." overall mag of pc applications (IJCA). ICNIC 2016.

[2] Dalal, N. Additionally, Triggs, B., "Histograms of orientated Gradients for Human Detection," IEEE pc Society appear on pc vision and test commonness, 2005, San Diego, CA, u.S. Of the us.

[3] D. G. Lowe, "object affirmation from close-by scale invariant aptitudes," in IEEE ICCV, 1999, vol. 2, p. 1150.

[4] N. Inoue and K. Shinoda, "Fast coding of feature vectors using neighborto-neighbor search," IEEE Trans. Pattern Anal. Mach. Intell., vol. 38, no. 6, pp. 1160-1184, 2016.

[5] J. Y. Choi, K. N. Plataniotis, and Y. M. Ro, "Using color local binary pattern features for face recognition," in Proc. 17th IEEE Int. Conf. Image Process. (ICIP), Sep. 2010, pp. 4541-4544.

[6] David G Lowe, "Distinctive Image Features from Scale-Invariant Keypoints", International Journal of Computer Vision, vol.50, No. 2, 2004, pp.91-110

[7] Herbert Bay, Tinne Tuytelaars and Luc Van Gool. "Speeded-up robust features (SURF)." Computer vision and image understanding, vol.110, No.3, 2008, pp.346-359.

[8] Ethan Rublee, Vincent Rabaud, Kurt Konolige and Gary Bradski, "ORB: and efficient alternative to SIFT or SURF", IEEE International Conference on Computer Vision,2011.

[9] E. Karami, S. Prasad, and M. Shehata, "Image Matching Using SIFT, SURF, BRIEF, and ORB: Performance Comparison for Distorted Images," in Proceedings of the 2015 Newfoundland Electrical and Computer Engineering Conference, St. John's, Canada, November,2015.

[10] Y. Bastanlar, A. Temizel and Y. Yardımc1, "Improved SIFT matching for image pairs with scale difference", Electron. Lett., vol. 46, no. 5, p. 346, 2010.

[11] Y. Cong, X. Chen and Y. Li, "Research on the SIFT Algorithm in Image Matching," AMM, vol. 121-126, pp.4656-4660,2011.

[12] B. Liao and H. Wang, "The Optimization of SIFT Feature Matching Algorithm on Face Recognition Based on BP Neural Network," $A M M$, vol. 743, pp. 359-364,2015.

[13] X. Qiao, "Research on the Algorithm of Image Matching Based on Improved SIFT," AMM, vol. 686, pp. 348-353,2014.

[14] F. Tian and Y. Yan, "A SIFT Feature Matching Algorithm Based on Semi-Variance Function," AMR, vol. 647, pp. 896-900,2013.

[15] H. Zhang and L. Cao, "An Image Matching Algorithm Based on SUSANSIFT Algorithm," $A M M$, vol. 325-326, pp. 1588-1592,2013.

[16] D. Nister and H. Stewenius, "Scalable recognition with a vocabulary tree," Proc. International Conference on Computer Vision and Pattern Recognition,Vol. 2, pp.2161-2168, 2006. 